\title{
O Papel da Inversão Sísmica no Processo de Caracterização de Reservatórios
}

\author{
Armando Zaupa Remacre, Rodrigo de Souza Portugal, Sérgio Sacani Sancevero, IGE/UNICAMP
}

Copyright 2004, SBGf - Sociedade Brasileira de Geofísica

Este texto foi preparado para a apresentação no I Simpósio de Geofísica da Sociedade Brasileira de Geofísica, São Paulo, 26-28 de setembro de 2004. Seu conteúdo foi revisado pela Comissão Tecno-científica do I SR-SBGf mas não necessariamente representa a opinião da SBGf ou de seus associados. E proibida a reprodução total ou parcial deste material para propósitos comerciais sem prévia autorização da SBGf.

\section{Resumo}

O processo de caracterização de reservatórios consiste na determinação tridimensional e quantitativa da estrutura e das propriedades petrofísicas de um campo de petróleo. A utilização do dado sísmico nesse processo tem se tornado cada vez mais comum, principalmente na determinação da forma e na identificação dos corpos que constituem o reservatório. Porém em alguns casos como na Bacia de Campos os reservatórios são constituídos por corpos com espessura subsísmica, ou seja, com espessura menor que o limite de resolução vertical da sísmica. Desse modo ao se utilizar o dado sísmico convencional de amplitude grandes erros podem ocorrer na estimativa do volume, pois nessas circunstâncias esse tipo de informação não permite um completo entendimento do reservatório. Para superar esse problema a maneira mais efetiva de se integrar o dado sísmico no processo de caracterização é através da construção de modelos de impedância acústica derivados do processo de inversão sísmica. O objetivo deste trabalho é mostrar através do modelo sintético de uma cunha, que é representativo de afinamentos normalmente encontrados em reservatórios turbidíticos de águas profundas, como a inversão do dado sísmico para a impedância acústica pode auxiliar o processo de caracterização de reservatórios. Outro objetivo é comparar os resultados obtidos através de dois método de inversão a inversão recursiva e o algoritmo denominado constrained sparse-spike. Ao final do trabalho pode-se ressaltar a utilização do processo de inversão sísmica, tanto na determinação da geometria dos reservatórios como também no cálculo de propriedades petrofísicas, e na construção de um modelo mais efetivo do reservatório onde as previsões de comportamento podem ser realizadas de maneira mais precisa.

\section{Introdução}

Para se otimizar o processo de entendimento dos reservatórios é necessário combinar as mediadas e conhecimentos geológicos, petrofísicos e geofísicos (Buiting et al., 1997). Contudo a integração das informações provenientes de diferentes áreas não é fácil de ser realizada na prática, devido principalmente às diferentes escalas de medidas e às diferentes origens da informação. A inversão do dado sísmico para a impedância acústica tem provado ser uma excelente ferramenta para estudos integrados.

A inversão do dado sísmico para a impedância acústica é um problema que os geofísicos têm se interessado por décadas (Latimer, et al., 2000). A vantagem de se utilizar a inversão sísmica em estudos relacionados com a caracterização de reservatórios e um dos motivos que tornou esse método vastamente utilizado na indústria de petróleo é que ele fornece do dado sísmico uma estimativa quantitativa da distribuição de vários parâmetros físicos que caracterizam a subsuperfície (Lailly, 1990).

A interpretação dos dados com o objetivo de encontrar ou investigar armadilhas estratigráficas que podem potencialmente constituir reservatórios é uma tarefa delicada e que requer uma análise cuidadosa do dado sísmico. A contribuição potencial da inversão sísmica é de tentar simplificar esse trabalho. Sabe-se que o dado sísmico representa uma propriedade de interface onde os eventos de reflexão são observados devido às mudanças relativas na impedância acústica de camadas de rochas adjacentes. As mudanças observadas na amplitude, contudo, não podem iniciar se a variação está relacionada com a litologia acima ou abaixo da interface. A impedância acústica é uma propriedade física da rocha, resultado do produto entre a velocidade e a densidade. Desse modo a impedância acústica é considerada uma propriedade de camada, enquanto que a amplitude sísmica é um atributo relacionado com as interfaces das camadas. Sendo a impedância acústica uma propriedade de camada ela simplifica a identificação litológica e estratigráfica e pode ser diretamente convertida para propriedades do reservatório como porosidade, tipo de fluido e espessura porosa (Chopra, et al., 2001).

Desse modo o objetivo principal desse trabalho é mostrar como a inversão sísmica para a impedância acústica pode auxiliar tanto o processo de interpretação como o processo de obtenção de propriedades petrofísicas. Para tanto são comparados os resultados obtidos a partir de dois métodos de inversão (inversão recursiva e a constrained sparse-spike inversion) de um modelo sintético de uma cunha. Esse modelo embora simples é representativo de certas estruturas normalmente encontradas em reservatórios como pinch-outs, afinamentos de corpos de areia e extremidades de estruturas canalizadas. A vantagem em se utilizar um modelo sintético em estudos de caracterização é o fato de que as interpretações são conclusivas, uma vez que, os resultados são facilmente validados com o modelo original. Outra vantagem é o fato de se poder utilizar o modelo sintético no processo de calibração e parametrização dos diferentes algoritmos de inversão. Ao final do trabalho pode-se analisar a resposta resultante 
dos dois algoritmos utilizados em termos da reprodução da geometria da estrutura modelada e da reprodução da impedância acústica. Com esses resultados pode-se concluir que a inversão sísmica tem um importante papel na interpretação sísmica, funcionando como uma poderosa ferramenta para otimizar o processo de caracterização de reservatórios.

\section{Metodologia}

O objetivo final do processo de inversão é reconstruir a impedância acústica em subsuperfície a partir dos dados sísmicos, dados de poços e outras informações a priori disponíveis. Essa reconstrução pode não ser realizada de maneira perfeita uma vez que a geologia em subsuperfície possui detalhes que não são imageados pela sísmica, devido ao limite de resolução vertical da informação. Para se obter um resultado satisfatório todos os dados disponíveis como a sísmica e os poços e todas as informações a priori como a wavelet e a estratigrafia, devem ser utilizados.

Independente do algoritmo utilizado o processo de inversão sísmica envolve quatro etapas principais, sendo elas: a construção de um modelo de subsuperfície, a extração da wavelet, a inversão propriamente dita e a união de traços.

A construção do modelo de subsuperfície determina a validade da modelagem final. É criado nessa etapa um volume sólido representativo da geologia da área em estudo. A partir dos perfis de impedância acústica nos poços e dos horizontes interpretados do dado sísmico é construído esse modelo de subsuperfície através da interpolação dos dados de poços ao longo de superfícies de correlação definidas pelos horizontes interpretados. A parte de baixa frequência do modelo gerado fornece uma boa estimativa da tendência da impedância acústica dependendo da distribuição dos poços e da qualidade da interpretação.

A variação de alta frequência da impedância acústica é extraída da refletividade sísmica. Esse processo é realizado deconvolvendo a wavelet como o objetivo de aumentar a banda de frequência nos altos valores aumentando desse modo a resolução.

A etapa de união dos traços é normalmente a última fase na construção do modelo de impedância, esse processo na teoria tem o objetivo de melhorar os resultados da inversão aumentando a banda de frequência. Um componente de baixa frequência do modelo de subsuperfície é extraído e unido com o dado resultante do processo de inversão.

A etapa de inversão sísmica propriamente dita pode ser realizada de diversas maneiras possíveis. Neste trabalho a inversão sísmica é realizada por meio de dois algoritmos. O primeiro método usado para se calcular a impedância acústica é a inversão recursiva. Talvez essa seja a técnica mais popular usada para se inverter o dado sísmico. A inversão recursiva é baseada na equação onde o coeficiente de reflexão é fornecido em termos da impedância acústica de camadas adjacentes, de acordo com:

$$
R C_{n}=\frac{\left[(\rho v)_{n+1}-(\rho v)_{n}\right]}{\left[(\rho v)_{n+1}+(\rho v)_{n}\right]} .
$$

Onde $R C_{n}$ é o coeficiente de reflexão na enésima camada e o produto $(\rho v)_{n+1}$ é a impedância acústica no enésimo intervalo. A Equação 1 pode ser reescrita para expressar $(\rho v)_{n+1}$ em termos de $(\rho v)_{n}$ e $R C_{n}$ :

$(\rho v)_{n+1}=(\rho v)_{n} \cdot\left[\frac{\left(1+R C_{n}\right)}{\left(1-R C_{n}\right)}\right]$.

Se o traço sísmico for visto como uma série de coeficientes de reflexão, então uma aplicação recursiva da Equação 2 fornece todas as impedâncias se $(\rho v)_{0}$ é conhecido. Para o traço sísmico ser exatamente igual à série de coeficientes de reflexão, alguns cuidados devem ser tomados no processamento do dado sísmico de modo que o dado final seja livre de efeitos que afetam o modelo.

O outro método utilizado para a obtenção da impedância acústica nesse trabalho é o chamado constrained sparsespike inversion, denominado inversão CSSI. Esse método cria um modelo de impedância acústica a partir de uma amplitude sísmica conhecida. Restrições são estabelecidas usando tendências geológicas observadas nos perfis de impedância acústica dos poços. Esse método de inversão assume que a sísmica usada é representativa da tendência geológica observada na impedância acústica. Esse processo de inversão é controlado por um conjunto de restrições baseadas numa informação a priori de um modelo geológico ou de perfis de poços. Essas restrições efetivamente limitam as potencias soluções reduzindo a não unicidade da resposta tendo assim um significado geológico e geofísico melhor. De maneira simplificada a inversão CSSI é baseada no algoritmo que resolve um problema de otimização restrito que pode ser escrito como:

$F=L_{1}(r)+\lambda L_{2}(s-d)+\alpha^{-1} L_{3}\left(\Delta z_{\text {tendencia }}\right)$.

Onde $F$ representa a função objetivo que deve ser minimizada. Os operadores, $L_{1}, L_{2}$ e $L_{3}$ são operações de deconvolução. Onde $L_{1}$ representa a soma total dos valores absolutos dos coeficientes de reflexão. O operador $L_{2}$ representa a diferença entre o dado sísmico (s) e o dado sintético (d) gerado para a modelagem da impedância e o termo $L_{3}$ representa a amarração com a tendência observada nos dados de poços. $O$ termo $\lambda$ fornece um peso à relação entre o dado sísmico e o sintético. Esse termo é importante para o processo e a sua definição controla o resultado. Se o valor de $\lambda$ for alto o termo (s-d) é enfatizado e o resultado será detalhado e com baixos resíduos. Um baixo valor de $\lambda$ enfatiza o termo da refletividade e o resultado terá poucos contrastes em impedância acústica com altos resíduos. O trabalho é selecionar um valor de $\lambda$, de modo que o modelo de impedância gerado não seja super detalhado mas que tenha um bom ajuste com a sísmica. 
O modelo sintético de uma cunha que representa certas situações estratigráficas encontradas em reservatórios é usado nesse trabalho para exemplificar os métodos de inversão descritos e mostrar as vantagens de se usar um modelo de impedância no processo de interpretação e caracterização de reservatórios.

\section{Resultados e Discussão}

A primeira etapa do trabalho consiste na geração do modelo sintético a ser utilizado. O modelo aqui usado consiste de uma cunha de baixa impedância representando uma camada de areia constituinte de um reservatório, encaixada num fundo de alta impedância representando folhelhos. O modelo foi preenchido com as propriedades velocidade e densidade com valores constantes e a partir da multiplicação de ambas as propriedades obteve-se o modelo original de impedância acústica. Esse modelo é mostrado na Figura 1.

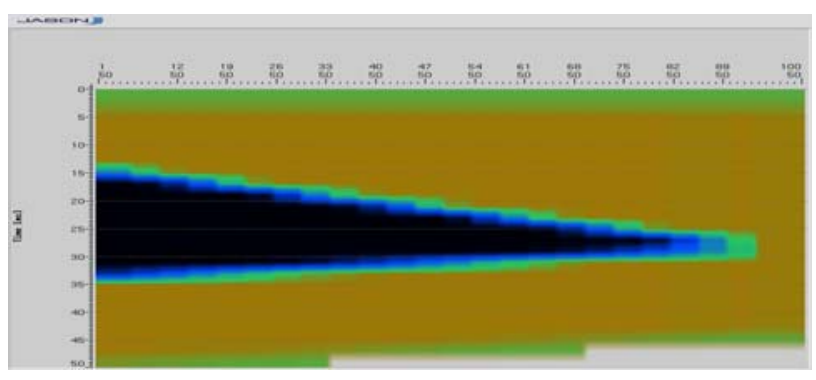

Figura 1 - Modelo de impedância original da cunha.

Após a construção do modelo, a próxima etapa consiste na geração do dado sísmico sintético. A sísmica sintética foi gerada através da modelagem convolucional, onde o traço sísmico é representado pela convolução entre a wavelet com a série de coeficientes de reflexão. Os coeficientes de reflexão são obtidos a parir do modelo de impedância. A wavelet utilizada neste trabalho é uma Ricker com frequência de $40 \mathrm{~Hz}$.

Na Figura 2 é apresentado o dado sísmico obtido pela modelagem convolucional.

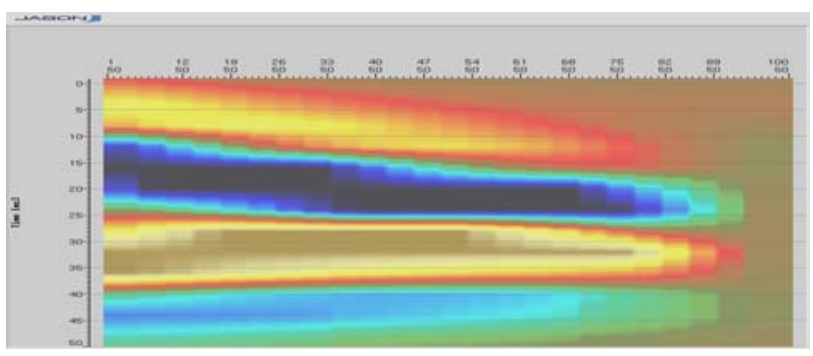

Figura 2 - Dado sísmico sintético relativo à cunha.

Pode-se observar a perda de resolução se comparado com o modelo original de impedância acústica. Essa perda de resolução se deve ao fato da cunha possuir uma espessura inferior ao limite de resolução vertical da sísmica e ainda apresenta um afinamento, ou seja, a espessura sofre uma redução gradativa provocando assim uma interferência entre os lobos laterais da wavelet. Pode-se observar também que a identificação da geometria acunhada é difícil de ser realizada no dado sísmico. Desse modo numa situação semelhante a aqui modelada seria impossível através do dado sísmico reproduzir a estrutura de interesse.

Com o objetivo de tentar recuperar a geometria da estrutura modelada foram realizadas as inversões sísmicas para a impedância acústica. $O$ primeiro método utilizado é a inversão recursiva. O resultado da inversão recursiva é apresentado na Figura 3.

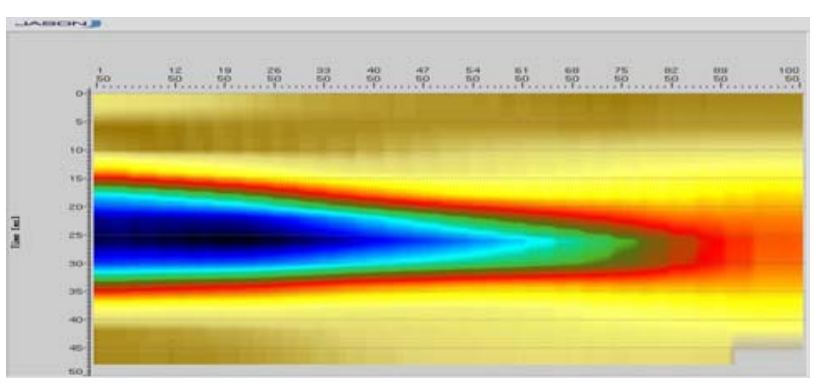

Figura 3 - Resultado da inversão recursiva para o modelo da cunha.

Pode-se observar na Figura 3 que a aplicação da inversão recursiva consegue recuperar um pouco da geometria da cunha, porém somente na região mais espessa, na porção mais fina da cunha grandes incertezas ainda persistem na delineação da estrutura acunhada. $O$ algoritmo fornece o resultado de maneira quase instantânea revelando desse modo uma das vantagens deste algoritmo, ou seja, a velocidade de processamento.

De modo a tentar aprimorar o resultado da inversão recursiva, q impedância acústica foi calculada também através da inversão CSSI. O resultado desta inversão é apresentado na Figura 4.

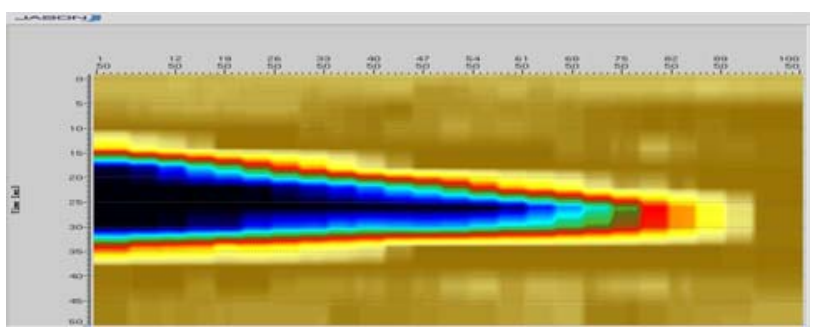

Figura 4 - Resultado da inversão CSSI para o modelo da cunha.

Observa-se no resultado apresentado na Figura 4 a quase completa delimitação da estrutura da cunha modelada. Isso se deve ao fato da integração das informações dos poços no processo de inversão adicionando as baixas freqüências e das deconvoluções adicionando as altas freqüências. Assim a resolução é aumentada e pode-se realizar interpretações mais precisas do que as feitas utilizando-se o dado sísmico e do resultado da inversão recursiva. Para enfatizar essas considerações foram extraídos dos volumes de impedâncias somente os valores relativos à cunha representando o corpo da cunha, os valores estão entre 5500 e 8400 g.m $/ \mathrm{cm}^{3}$.s. As seções com esses valores são mostradas na Figura 5. 


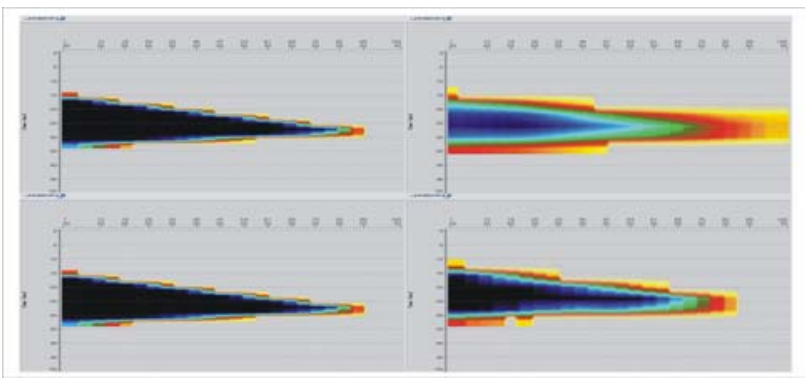

Figura 5 - Comparação entre o modelo original e a inversão recursiva (topo) e o modelo original e a inversão CSSI (base) com os valores relativos à cunha.

Através das comparações realizadas na Figura 5 pode-se observar que além de recuperar a geometria da cunha com maior precisão, a propriedade petrofísica também é melhor definida quando se utiliza a inversão CSSI. No caso da inversão recursiva a geometria é recuperada para as espessuras maiores, porém pode-se observar que a reprodução da propriedade petrofísica não é realizada de maneira satisfatória. Essas observações são ainda comprovadas quando se calcula a diferença entre o modelo original de impedância acústica e os resultados da inversão, esse resultado é mostrado na Figura 6.

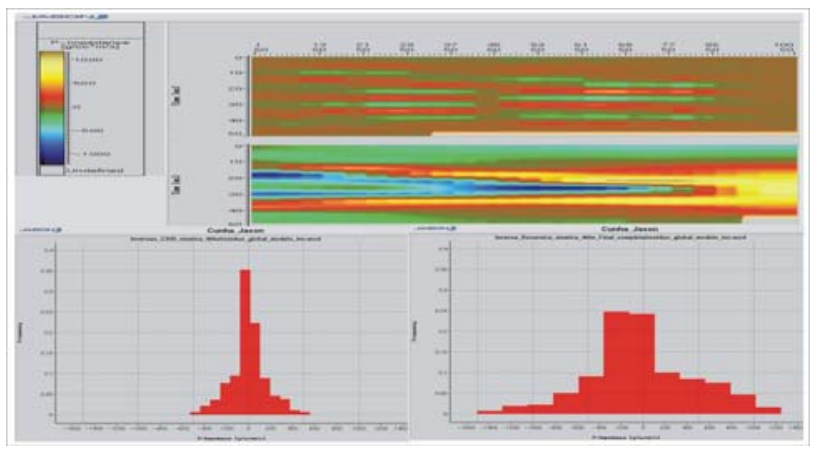

Figura 6-Diferença entre o modelo original e os resultados das inversões CSSI e recursiva respectivamente juntamente com os histogramas das diferenças.

Pode-se observar na Figura 6 que o resultado da inversão CSSI apresenta uma diferença menor com relação ao modelo original, com grande parte dos valores com resíduo zero. No caso da inversão recursiva pode-se notar que na parte interna da cunha as diferenças são negativas indicando que a inversão fornece valores maiores do que os originais, já na parte externa a diferença possui valores positivos indicando que a inversão resulta em valores menores que os originais, desse modo entende-se o comportamento da inversão recursiva em casos de afinamento de camadas. O histograma da diferença com relação à inversão recursiva apresenta uma maior variância indicando uma grande incerteza no caso de se prever a espessura de camadas com espessura subsísmica.

\section{Conclusões}

O exemplo mostrado nesse trabalho demonstra claramente a superioridade de se utilizar a inversão sísmica no processo de caracterização de reservatórios, principalmente em casos onde os corpos que constituem o reservatório possuem espessuras inferiores ao limite de resolução vertical da sísmica. O modelo sintético aqui desenvolvido, embora simples é suficiente para mostrar as vantagens de se utilizar o processo de inversão sísmica. Além disso esse modelo é representativo de algumas situações estratigráficas encontradas em reservatórios, como pinch-outs, afinamento dos corpos de areia e extremidades de estruturas canalizadas. O método recursivo de inversão conseguiu resolver a geometria do modelo somente nas espessuras maiores, nas regiões mais finas do modelo incertezas grandes ocorrem principalmente na identificação do final da estrutura. A vantagem de se utilizar esse método está no seu reduzido tempo de processamento, desse modo em casos reais pode-se utilizar a inversão recursiva para promover uma primeira melhora na interpretação no caso de camadas espessas mesmo que não sejam mapeadas pela sísmica convencional. Já o método de inversão CSSI mostrou resultados muito superiores tanto na identificação geométrica do modelo como na reprodução da distribuição da propriedade petrofísica. Embora o tempo computacional seja maior isso é compensado pela melhoria observada nos resultados e principalmente na redução de incertezas ao se interpretar as regiões mais finas do modelo e identificar o fim da estrutura. Finalmente pode-se concluir que a inversão sísmica para a impedância acústica se mostrou uma poderosa ferramenta para auxiliar o processo de caracterização de reservatório. Desse modo ao se aplicar essa metodologia na exploração e desenvolvimento de reservatórios, devido ao aumento da resolução, a imagem mais consistente com a realidade e a integração de todas as informações disponíveis, modelos mais precisos do reservatório podem ser gerados otimizando assim a localização de novas zonas produtoras e principalmente à previsão do comportamento do campo.

\section{Referências}

Buiting, J. J. M., Bacon, M., 1997. Using Geophysical, Geological, and Petrophysical Data to Characterize Reservoirs in the North Sea. $5^{\text {th }}$ Conference on Petroleum Geology of NW Europe

Chopra, S., Kuhn, O., 2001. Seismic Inversion. CSEG Recorder, vol. 26, n.1: 12-14.

Lailly, P., 1990. Blind Tests of Inversion Packages for Stratigraphic Interpretaion. Revue de L'Insitut Français du Pétrole. In: Proceedings of Workshop on "Practical Aspects of Seismic Inversion, vol. 45 n.3:301-326.

Latimer, R. B., Davison, R., Riel, van P., 2000. An Interpreter's Guide to understanding and Working with Seismic-Derived Acoustic Impedance Data. The Leading Edge, March 2000: 242-256. 